\title{
COMMENTARY
}

\section{Blood pressure variability: does speed matter?}

\author{
Praveen Veerabhadrappa \\ Hypertension Research (2013) 36, 1035-1036; doi:10.1038/hr.2013.114; published online 12 September 2013
}

$\mathrm{B}$ lood pressure (BP) is dynamic. Conventional assessment of BP only provides a snapshot of continuous fluctuations. BP fluctuation, also known as BP variability, has been identified as an important independent cardiovascular risk factor and is associated with both the prevalence and severity of target-organ damage, regardless of the BP levels in different populations. ${ }^{1-5}$ Fluctuations in BP range from fast changes lasting only a few seconds, to slower and more prolonged variations over minutes, hours or even days or months. ${ }^{6}$

Conventional measures of BP variability, such as the s.d. or the coefficient of variation of the average ambulatory systolic BP, have been challenged due to their limited predictive power. ${ }^{7}$ The s.d. of BP variability is a less precise indicator of true $\mathrm{BP}$ dynamics, as it only reflects the dispersion of $\mathrm{BP}$ measurements around the mean and does not account for the order or time rate of $\mathrm{BP}$ variation (a measure of the speed of $\mathrm{BP}$ fluctuations) in BP measurements obtained over a 24 -h period. ${ }^{8}$ In addition, the s.d. of BP variability could be influenced by physiological factors, such as nocturnal BP, as well as by some environmental factors. These factors suggests a need to use indices other than the s.d. because the same s.d. (or the coefficient of variation) value may be associated with completely different patterns of BP variability. ${ }^{9}$

To address these challenges and to both improve the assessment of BP variability and obtain quantifiable information on the speed of changes in systolic BP, many novel techniques are evolving. ${ }^{10}$ To quantify the speed of changes in BP variability, the average real variability (ARV index) of $\mathrm{BP}$

Dr $\mathrm{P}$ Veerabhadrappa is at the Department of Exercise Science, Shippensburg University, Shippensburg, PA, USA

E-mail: pveer@ship.edu or praveenv@temple.edu variability was proposed by Mena et al. ${ }^{8}$ ARV is quantified by calculating the mean of the differences between two successive BP measurements taken during 24-h ambulatory blood pressure monitoring (ABPM). In a large cohort study, ARV was a better predictor of cardiocerebrovascular events and cardiovascular mortality than some of the more conventional measures of $\mathrm{BP}$ variability. ${ }^{11}$ In 2005, Zakopoulos et al. ${ }^{12}$ proposed the 'time rate of BPV' to measure the speed and direction of systolic BP value change. The 'time rate of BPV' was derived from 24-h ABPM and was defined as the first derivative of the systolic BP values against time. Manios et al. ${ }^{13}$ used this index to quantify short-term changes in systolic BP in normotensive subjects with suspected coronary artery disease, and the authors found that the 'time rate of BPV' was more pronounced in subjects with coronary atherosclerotic lesions when compared with controls with normal angiography. However, the use of ABPM for estimating $\mathrm{BP}$ variability has been challenged because of the length of the spacing of BP measurements. ${ }^{9,14}$ Mancia et al. ${ }^{15}$ showed that short-term changes in the systolic BP and quantification of the speed of systolic BP changes over short time intervals were significantly greater in hypertensive subjects than in normotensive individuals; even more important were the differences in the slope of the rapid and short-duration changes in systolic BP.

Owing to its intricate nature, a precise and detailed assessment of short-term BP variability can be obtained from the analysis of continuous, beat-to-beat $\mathrm{BP}$ recordings. In the past, continuous BP recordings were assessed invasively. Recently, advanced noninvasive devices that measure finger $\mathrm{BP}$ on a beat-by-beat basis have been developed, including the Finapres monitor (Finapres OHMEDA, Englewood, CO, USA) followed by the Portapres (Portapres, Finapres Medical Systems, Amsterdam, The Netherlands) and the Finometer device (Finometer, Finapres Medical Systems); these devices have made non-invasive continuous BP monitoring possible in ambulatory conditions over $24 \mathrm{~h}$. The advent of these non-invasive techniques in combination with novel mathematical approaches for assessing BPV have made beat-to-beat assessment feasible within the clinical setting. ${ }^{16}$

In this issue of Hypertension Research, van Gestel et al. ${ }^{17}$ investigate for the first time the degree and speed of fluctuations in the systolic BP in a group of subjects with a heterogeneous cardiovascular risk profile. This study utilized non-invasively obtained continuous hemodynamic measurements of beat-to-beat BP recordings over 5 min using a Finometer device. By calculating the slopes of the oscillatory fluctuations in the systolic $\mathrm{BP}$ for different interbeat intervals, the authors quantified the speed of the systolic BP (vSBP) changes. They found that the vSBP in subjects considered at high cardiovascular risk was significantly higher than in subjects with no or low cardiovascular risk. Interestingly, they found that the degree of BP variability as quantified by s.d. was not associated with cardiovascular risk. Taken together, these findings may provide evidence that the speed of changes in systolic BP rather than the amount of BPV may have a crucial role in cardiovascular disease. Of course, given the modest sample size of the study cohort $(n=122)$, these cross-sectional study findings will need to be confirmed in much larger clinical trials. Nonetheless, they do shed light on the potential dynamics of BP variability in subjects with cardiovascular disease.

Although the findings presented by van Gestel et al. are interesting and certainly could suggest that BP variability enthusiasts should be paying attention to not only the 
degree of BP variations but also the speed of systolic BP variations, their findings perhaps raise more questions than answers. First, what are the mechanisms by which fluctuations in systolic BP contribute to the occurrence or progressive deterioration of cardiovascular disease? Second, what diagnostic procedures and therapeutic steps should be performed to manage BPV? Third, is there a clinical advantage to addressing high systolic BP fluctuations? In answer to some of these vital questions, steep BP variations could increase the oscillatory shear stress in the vessel wall of medium and large arteries. This enhances the traumatic effects of intravascular pressures on the vessel wall, which may ultimately result in the pathogenesis of cardiovascular disease. ${ }^{13}$ Unfortunately, measures of vascular structure or function were not assessed in the present study; thus, the precise mechanism remains to be elucidated. BP variability has increasingly been recognized as a novel risk factor that can predict not only primary but also recurrent stroke risk and provide information that complements the mean BP levels. ${ }^{18}$ Apart from the magnitude of $\mathrm{BP}$ elevation, it is increasingly being recognized that the time rate of BP variation, a relatively new index of BP variation, offers additional prognostic significance for target-organ damage. ${ }^{19}$

In conclusion, whether we should consider assessing the speed of $\mathrm{BP}$ fluctuations as an additional measure of BP variation still remains unclear and the available evidence is not strong enough to support this practice. However, it has been proposed that the future of BP variability assessment will be to develop devices that measure beat-to-beat ambulatory BP non-invasively and that could be used in large and long-term studies in a cost-effective manner to detect even subtle differences in the magnitude and type of BP variations. ${ }^{9}$ It may also be possible to assess the speed of the BP changes during the frequent pressor and depressor episodes that occur spontaneously over $24 \mathrm{~h}$ on a large scale. ${ }^{9}$ In addition, it has been shown that BP changes faster throughout the day and night in hypertensive subjects than in normotensive subjects, ${ }^{15}$ but no data are available on whether the potentially greater traumatic effect of faster BP changes on the heart and the vessel wall favors organ damage and increases the risk of cardiovascular events. ${ }^{9}$ Further, prospective work is needed to elucidate whether altering BP variability will improve cardiovascular outcomes. Finally, longitudinal studies on beat-to-beat systolic BP measurements in different cohorts of subjects with varied cardiovascular risk profiles are needed to assess its clinical usefulness.

1 Parati G, Faini A, Valentini M. Blood pressure variability: its measurement and significance in hypertension. Curr Hypertens Rep 2006; 8: 199-204.

2 Mancia G, Ferrari A, Gregorini L, Parati G, Pomidossi G, Bertinieri G, Grassi G, Zanchetti A. Blood pressure variability in man: its relation to high blood pressure, age and baroreflex sensitivity. Clin Sci (Lond) 1980; 59 (Suppl 6), 401s-404s.

3 Mancia G, Parati G, Hennig M, Flatau B, Omboni S, Glavina F, Costa B, Scherz R, Bond G, Zanchetti A. Relation between blood pressure variability and carotid artery damage in hypertension baseline data from the European Lacidipine Study on Atherosclerosis (ELSA). J Hypertens 2001; 19 1981-1989.

4 Veerabhadrappa P, Diaz KM, Feairheller DL, Sturgeon KM, Williamson S, Crabbe DL, Kashem A Ahrensfield D, Brown MD. Enhanced blood pressure variability in a high cardiovascular risk group of African Americans: FIT4Life Study. J Am Soc Hypertens 2010; 4: 187-195.

5 Diaz KM, Veerabhadrappa P, Kashem MA, Feairheller DL, Sturgeon KM, Williamson ST, Crabbe DL, Brown MD. Relationship of visit-to-visit and ambulatory blood pressure variability to vascular function in African Americans. Hypertens Res 2012; 35: 55-61.

6 Parati G, Bilo G, Vettorello M, Groppelli A, Maronati A, Tortorici E, Caldara G, Mancia G. Assessment of overall blood pressure variability and its different components. Blood Press Monit 2003; 8: 155-159.

7 Pierdomenico SD, Di NM, Esposito AL, Di MR, Ballone E, Lapenna D, Cuccurullo F. Prognostic value of different indices of blood pressure variability in hypertensive patients. Am J Hypertens 2009; 22 842-847.

8 Mena L, Pintos S, Queipo NV, Aizpurua JA, Maestre G Sulbaran T. A reliable index for the prognostic significance of blood pressure variability. J Hypertens 2005; 23: 505-511.

9 Mancia G. Short- and long-term blood pressure variability: present and future. Hypertension 2012; 60 512-517.

10 Bilo G, Giglio A, Styczkiewicz K, Caldara G, KaweckaJaszcz K, Mancia G, Parati G. How to improve the assessment of 24-h blood pressure variability. Blood Press Monit 2005; 10: 321-323.

11 Hansen TW, Thijs L, Li Y, Boggia J, Kikuya M, Bjorklund-Bodegard K, Richart T, Ohkubo T, Jeppesen J, Torp-Pedersen C, Dolan E, Kuznetsova T, Stolarz-Skrzypek K, Tikhonoff V, Malyutina S, Casiglia E, Nikitin $Y$, Lind L, Sandoya E Kawecka-Jaszcz K, Imai Y, Wang J, Ibsen H, O'Brien $\mathrm{E}$, Staessen JA. Prognostic value of reading-to-reading blood pressure variability over $24 \mathrm{~h}$ in 8938 subjects from 11 populations. Hypertension 2010; 55 1049-1057.

12 Zakopoulos NA, Tsivgoulis G, Barlas G, Papamichael C, Spengos K, Manios E, Ikonomidis I, Kotsis V, Spiliopoulou I, Vemmos K, Mavrikakis M, Moulopoulos SD. Time rate of blood pressure variation is associated with increased common carotid artery intimamedia thickness. Hypertension 2005; 45: 505-512.

13 Manios E, Stamatelopoulos K, Tsivgoulis G, Barlas G, Koroboki E, Tsagalis G, Michas F, Vemmos $K$ Zakopoulos $\mathrm{N}$. Time rate of blood pressure variation: a new factor associated with coronary atherosclerosis. J Hypertens 2011; 29: 1109-1114.

14 Bilo G, Parati G. Rate of blood pressure changes assessed by $24 \mathrm{~h}$ ambulatory blood pressure monitoring: another meaningful index of blood pressure variability? J Hypertens 2011; 29: 1054-1058.

15 Mancia G, Parati G, Castiglioni P, Tordi R, Tortorici E, Glavina F, Di RM. Daily life blood pressure changes are steeper in hypertensive than in normotensive subjects. Hypertension 2003; 42: 277-282.

16 Parati G, Ongaro G, Bilo G, Glavina F, Castiglioni P, Di RM, Mancia G. Non-invasive beat-to-beat blood pressure monitoring: new developments. Blood Press Monit 2003; 8: 31-36.

17 van Gestel AJR, Camen G, Clarenbach CF, Sievi N, Rossi VA, Kohler M. Quantifying the speed of fluctuations in systolic blood pressure. Hypertens Res 2013; 36: 1039-1044.

18 Rothwell PM. Does blood pressure variability modulate cardiovascular risk? Curr Hypertens Rep 2011; 13: 177-186.

19 Zis P, Vemmos K, Spengos K, Manios E, Zis V, Dimopoulos MA, Zakopoulos N. Ambulatory blood pressure monitoring in acute stroke: pathophysiology of the time rate of blood pressure variation and association with the 1-year outcome. Blood Press Monit 2013; 18: 94-100. 